\title{
Qualitätssicherung - es wird konkreter, aber nicht leichter
}

\section{Christoph Röder}

Institutsleiter a. i, MEM

Forschungszentrum, Institut für Evaluative Forschung in der Orthopädie, Universität Bern
Korrespondenz:

PD Dr. med

Christoph Röder, MPH

MEM Forschungszentrum

Institut für Evaluative Forschung in der Orthopädie

Universität Bern

Stauffacherstrasse 78

CH-3014 Bern

Tel. 0316315932

Fax 0316315960

christoph.roeder[at]

memcenter.unibe.ch
Der schon fast zum Unwort verkommene Begriff der Qualitätssicherung (QS) geistert seit Jahren durch das schweizerische und andere Gesundheitssysteme. Während lange Zeit Einzelinitiativen auf Ebene Praxis, Abteilung oder Klinik in mehr oder weniger unverbindlichem Rahmen stattfanden, haben ökonomische Zwänge, politische Gegebenheiten und neue Strömungen in der Wissenschaft der QS zu einer nationalen und verbindlicheren Dimension verholfen.

Pünktlich zu den Meldungen von gefährlichen Ionenkonzentrationen im Blut von Trägern der Metall-Metall-Hüftprothesen aus Frankreich wurde in der Schweiz - nach langen Jahren der Vorbereitung - das SIRIS Implantatregister für Hüft- und Knieprothesen unter der Schirmherrschaft des ANQ in Kooperation mit der Schweizerischen Gesellschaft für Orthopädie und Traumatologie SGOT aus der Taufe gehoben. Der Skandal um die PIP-Brustimplantate verhilft der Diskussion um das seit 2009 bestehende und bis dato freiwillige Implantatregister der Schweizerischen Gesellschaft für Plastische und Rekonstruktive Chirurgie zu neuer Aktualität und Frage nach verpflichtender Teilnahme. Dazu haben sich z. B. die schweizerischen Nephrologen in einem neuen Vertrag mit den Kostenträgern verpflichtet. Jeder Dialysepatient wird zukünftig mindestens einmal jährlich mit einem minimalen, der übergeordneten EU-Arbeitsgruppe konformen Datensatz im SRRQAP, dem Swiss Renal Registry and Quality Assessment Program, dokumentiert. Schliesslich hat die Schweizerische Gesellschaft für Ophtalmologie Ende 2011 ihr Pilotprojekt eines Kunstlinsenregisters beendet und plant eine definitiven Start für den Spätsommer 2012. Es wird offensichtlich, dass die Qualitätsmessung und Sicherung vor allem bei teuren Therapien, bei denen häufig Implantate im Spiel sind, auf dem Weg der definitiven Umsetzung ist. Dies ist jedoch kein unumstösslicher Trend, denn auch die Spitex entwickelt derzeit ihr landesweites Pflegeregister zur Erforschung und Sicherung der Behandlungsqualität und deren Ergebnisse, den sogenannten Outcomes.

Medizinregister liegen im Trend. Obschon in der Schweiz dank Registerpionier Maurice E. Müller (MEM) seit über 35 Jahren in vor allem orthopädischer Anwendung, haben sich die Beobachtungen medizinischer Versorgung in der Alltagssituation und ihre zentralisierte Dokumentation nur langsam als anerkannte wissenschaftliche Methode durchgesetzt. Einer der Hauptgründe für diese Verzögerung war die evidenzbasierte Medizin, die in den letzten zehn Jahren den Fokus vor allem auf die interne Validität medizinischer Studien, und damit auf ihre methodologische Güte gerichtet hat. Dabei hat ausgerechnet der Vater der modernen Outcomeforschung, Archie Cochrane, in seinen Random Reflections on Health Services bereits 1972 den Unterschied zwischen randomisierten kontrollierten Studien, welche die Wirksamkeit einer Behandlung in einem künstlich optimierten Studiensetting untersuchen, und Beobachtungsstudien, welche die Zweckmässigkeit von Behandlungen in der klinischen Routine betrachten, herausgestellt. Letztere tragen bei zugegebenermassen begrenzter interner Validität vor allem eine hohe externe Validität, d. h. Übertragbarkeit auf die Allgemeinheit und sind damit für die tatsächliche Evaluation des Potentials einer Therapie von grosser Bedeutung. Dank der ungebrochenen Überzeugung des Institutsgründers MEM und seiner Nachfolger erfreut sich das seit 2003 an der Uni Bern beheimatete Institut für Evaluative Forschung in der Medizin einer stetig wachsenden Registerklientel auf seinem speziell dafür entwickelten MEMdoc Portal. Es ist daneben einer der Ausrichter des Nationalen Symposiums für Qualitätsmanagement im Gesundheitswesen (siehe Kasten).

Die Konkretisierung all dieser QS-Bemühungen ist zu begrüssen, doch ob Pilotstudie oder tatsächliche Umsetzung, Ernüchterung tritt oft dann ein, wenn bemerkt wird, dass aus administrativen oder medizinischen Routinedaten meist nicht die für die Qualitätsmessung benötigten Informationen zu gewinnen sind. Seien die erforderlichen Datensätze noch so klein, Zusatzaufwand muss betrieben werden, Prozesse geändert und Ressourcen aloziiert, ggfs. muss sich gar die gesamte «Kultur» einer Abteilung oder Klinik anpassen bzw. neu definieren. Hinzu kommen Medienbrüche, die viele nationale Projekte mit sich bringen. Aus Mangel an Schnittstellen mit z.B. zentralen Registerdatenbanken, oder auch aus Mangel an Finanzkraft, solche Schnittstellen aufseiten der Klinik zu programmieren, müssen die QSDatensätze in ein «externes», also nicht spitaleigenes Klinikinformationssystem eingegeben werden. Auf der anderen Seite ist ein zentraler Datenpool für QS-Projekte unbedingt zu befürworten, da nur so ein Benchmark, also ein aus landesweiten Daten errechneter Durchschnitts- und damit Referenzwert für bestimmte Indikatoren erarbeitet und zum Vergleich herangezogen werden kann. Der Mangel an Schnittstellen wird erwartungsgemäss mit zunehmender 
Abbildung 1

Modell der Versorgungs-/Gesundheitsforschung.

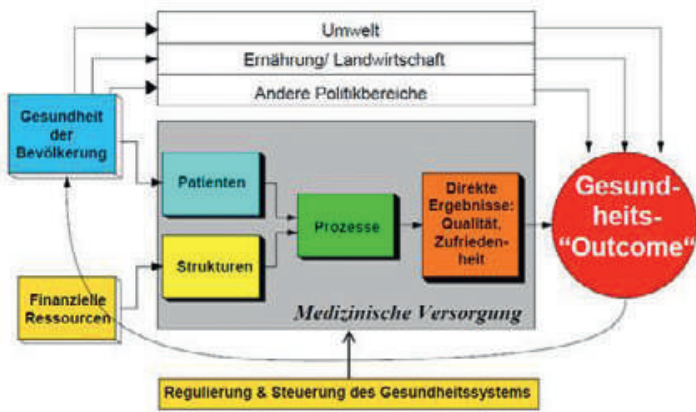

Reife der QS-Projektlandschaft in der Schweiz zurückgehen, da diese sich als nachhaltige Investitionen mit Perspektiven erweisen werden.

Neue Komplexität, aber auch Rückenwind erhält die Qualitätsdiskussion nicht nur wegen leerer Kassen und Pressemeldungen über gefährliche Implantate; die vielen eng mit QS verbundenen Aspekte der Outcomeforschung, der evidenzbasierten Medizin, der Nachverfolgung von Implantaten und der Qualitätsforschung sind in einer neuen, zunehmend an Bedeutung und Bekanntheit gewinnenden wissenschaftlichen Disziplin vereinigt: der Versorgungsforschung als Subdisziplin der Gesundheitssystemforschung.

Die Versorgungsforschung ist entstanden, weil sich die Lage im Gesundheitswesen zunehmend dadurch auszeichnet, dass den vielfältigen Problemen der Kranken- und Gesundheitsversorgung keine tragfähigen Problemlösungen gegenüberstehen. Die grossen Akteursgruppen in diesem Feld Praktiker, Politiker und Wissenschaftler - haben bisher keine schlüssige Antwort auf die drängende Frage gefunden, wie die ökonomischen und qualitätsbezogenen Probleme im Gesundheitswesen ge- löst werden können. Dies liegt zum einen an der Komplexität der Versorgungsprobleme, zum anderen aber auch an der begrenzten Problemlösungskapazität des Gesundheitswesens. Eine der zentralen Ursachen für die mangelnde Problemverarbeitungskapazität ist, dass es an Daten und Wissen über die Versorgungssituation und ihre «inneren» Zusammenhänge mangelt. An diesem Punkt setzt die Versorgungsforschung an. Das Ziel der Versorgungsforschung ist, grundlegendes und anwendungsnahes Wissen über die Praxis der Kranken- und Gesundheitsversorgung zu generieren und der Öffentlichkeit zur Verfügung zu stellen.

Die Versorgungsforschung kann definiert werden als ein fachübergreifendes Forschungsgebiet, das die Kranken- und Gesundheitsversorgung und ihre Rahmenbedingungen beschreibt und kausal erklärt, zur Entwicklung wissenschaftlich fundierter Versorgungskonzepte beiträgt, die Umsetzung neuer Versorgungskonzepte begleitend erforscht und die Wirksamkeit von Versorgungsstrukturen und -prozessen unter Alltagsbedingungen evaluiert. Gegenstand der Versorgungsforschung ist die «letzte Meile» des Gesundheitssystems. Unter dem Begriff der letzten Meile des Gesundheitssystems ist die konkrete Kranken- und Gesundheitsversorgung in den Krankenhäusern, Arztpraxen und sonstigen Gesundheitseinrichtungen $\mathrm{zu}$ verstehen, in deren Rahmen die entscheidenden Versorgungsleistungen zusammen mit dem Patienten erbracht werden.

Die aktuelle und zukünftige Qualitätsdiskussion und Umsetzung der QS wird von dieser jungen fachübergreifenden Wissenschaft entscheidend mitgeprägt werden. Sie macht die QS durch Einordnung in ein neues System von Modellen, Konzepten und Interaktionen griffiger und verständlicher, aber auch komplexer.

\section{Nationales Symposium für Qualitätsmanagement im Gesundheitswesen 15. Mai 2012 - Auditorium Ettore Rossi, Inselspital, Bern:}

\section{Qualitätsmanagement - vom «Schwarzen Peter» zum Wettbewerbsfaktor}

Qualitätsmanagement - ein zentraler und oft verwendeter Begriff in den aktuellen Diskussionen im Gesundheitswesen. Qualitätsmanagement ist ein Ansatz, ein Konzept aber auch eine Haltung, modern und vorwärtsgerichtet, wird jedoch auch gerne und schnell mit Bürokratie und Zusatzaufwand verknüpft. Trotz der Wichtigkeit des Themas herrscht auf übergeordneter Ebene so etwas wie Stillstand vor. Die Verantwortung und anstehende Entscheide werden wie der «Schwarze Peter» hin- und hergeschoben: vom Bund zu den Versicherern, weiter zu den Leistungserbringern und zurück. Es ist nicht erstaunlich, dass die grossen Würfe bislang ausgeblieben sind und immer wieder Resignation aufkommt.

Erfreulicherweise brachten strukturelle Veränderungen Bewegung ins Gesundheitssystem. Qualitätsmanagement und die Qualität werden plötzlich zu einem bedeutenden Wettbewerbs- faktor und erreichen möglicherweise den Stellenwert, der lange angestrebt wurde. Das nationale Symposium 2012 setzt sich mit diesen Fragen auseinander. Experten aus dem In- und Ausland zeigen Lösungsvorschläge und erörtern die zentralen Fragen. Sie beleuchten den Themenkreis aus verschiedenen praktischen und theoretischen Perspektiven.

Auch dieses Jahr wird dank der grosszügigen Unterstützung durch die FMH im Rahmen des Nationalen Symposiums der Swiss Quality Award verliehen.

Informationen zum Symposium und Anmeldungen unter www. qmsymposium.ch Informationen zum Swiss Quality Award unter www.fmh.ch 